\title{
Generative adversarial network- based photoacoustic image reconstruction from bandlimited and limited-view data
}

Kalloor Joseph, Francis, Arora, Aayush, Kancharla, Parimala, Kuniyil Ajith Singh, Mithun, Steenbergen, Wiendelt, et al.

Francis Kalloor Joseph, Aayush Arora, Parimala Kancharla, Mithun Kuniyil Ajith Singh, Wiendelt Steenbergen, Sumohana S. Channappayya, "Generative adversarial network-based photoacoustic image reconstruction from bandlimited and limited-view data," Proc. SPIE 11642, Photons Plus Ultrasound: Imaging and Sensing 2021, 1164235 (5 March 2021); doi: $10.1117 / 12.2577750$ 


\title{
Generative Adversarial Network-based Photoacoustic Image Reconstruction from Bandlimited and Limited-view Data
}

\author{
Kalloor Joseph Francis ${ }^{\mathrm{a}^{\dagger}}$, Aayush Arora ${ }^{\mathrm{b}^{\dagger}}$, Parimala Kancharla ${ }^{\mathrm{b}}$, Mithun Kuniyil Ajith Singh ${ }^{\mathrm{c}}$, \\ Wiendelt Steenbergen ${ }^{\mathrm{a}}$, and Sumohana S. Channappayya ${ }^{\mathrm{b}}$ \\ ${ }^{a}$ Biomedical Photonic Imaging Group, Technical Medical Center, University of Twente, The \\ Netherlands \\ ${ }^{\mathrm{b}}$ Department of Electrical Engineering, Indian Institute of Technology Hyderabad, 502285, \\ India \\ ${ }^{c}$ Research and Business Development Division, Cyberdyne Inc., Cambridge Innovation Center, \\ 3013 AK Rotterdam, The Netherlands
}

\begin{abstract}
Ultrasound transducers used in photoacoustic imaging are bandlimited and have a limited detection angle, which degrades the reconstructed image quality. One way to address this problem is to have transducers with multiple frequency bands with acquisition around the sample. This approach is expensive and it is not feasible for systems with a handheld probe using a linear transducer array. In this work, we aim to develop a deep learning method for photoacoustic reconstruction from bandlimited and limited-view data. We have developed a Generative Adversarial Networks (GANs)-based framework conditioned with a photoacoustic measurement for image reconstruction. In this way, the transducer used in the measurement can be incorporated and the generator trying to compensate for the limited data problem. We have developed the model for a handheld photoacoustic system using a linear transducer array with 128 elements having a center frequency of $7 \mathrm{MHz}$ and $-6 \mathrm{~dB}$ bandwidth from 4-10 MHz. We trained the network using simulated blood vessel images and tested it on in vivo measurements from the human forearm. We have compared the reconstructed images using the proposed method with the time-reversal on simulated data for detection using a bandlimited and directional transducer and compared it using the ground truth. Further, we compare our results to the in vivo images from the system which uses a delay and sum algorithm. The results from both simulations and experiments show that the proposed approach can remove bandlimited and limited view artifacts and can achieve a better image quality.
\end{abstract}

Keywords: Photoacoustic imaging, deep learning, generative adversarial network, artifact removal, image reconstruction, convolutional neural networks

\section{INTRODUCTION}

Photoacoustic (PA) imaging is an emerging modality for functional and molecular imaging of deep tissue. In PA imaging pulsed light induces thermoelastic expansion, resulting in acoustic waves from optical absorbers in the tissue. ${ }^{1}$ Thus PA imaging enables optical contrast at ultrasound resolution. The acoustic signals can be detected using ultrasound (US) transducers; which is an added advantage of PA imaging that we can perform conventional US imaging and co-registered the images for structural information. ${ }^{2}$ An obvious step is to convert conventional US systems to combined PA and US imaging systems by integrating a light source. This approach can find faster translation and wide acceptance in the clinical setting as it can be easily incorporated into the existing clinical practice. However, this approach comes with a limitation. Most of the commercially available US transducers are band-limited and has a limited opening angle for detection. However, PA signals are broadband and propagate in all directions. Detecting PA signal with a band-limited linear transducer array introduces artifacts. There is

Further author information:

* K. J. Francis.: E-mail: f.kalloorjoseph@utwente.nl, Telephone: +31534891559

$\dagger$ These authors contributed equally

Photons Plus Ultrasound: Imaging and Sensing 2021, edited by Alexander A. Oraevsky

Lihong V. Wang, Proc. of SPIE Vol. 11642, 1164235 - (c) 2021 SPIE

CCC code: $1605-7422 / 21 / \$ 21 \cdot$ doi: $10.1117 / 12.2577750$

Proc. of SPIE Vol. 11642 1164235-1 
a need to overcome this limitation in PA imaging for more practical applications.

The hardware-based solution involves tomographic imaging using multiple transducers to detect the PA signal from all the directions and the use of custom developed broadband transducers. ${ }^{3-5}$ Both these approaches come with a high cost. Hence, software-based solutions are widely attempted to enhance PA signal and to remove the artifacts. ${ }^{6}$ Model-based reconstruction methods were explored for this purpose. ${ }^{7}$ In recent years deep learningbased methods have shown promise in this aspect. ${ }^{8-12}$ Generative Adversarial Networks (GANs) were also used to address this problem where the authors used learning from time-reversal based reconstruction to the ground truth. ${ }^{13}$

In this work, we investigated the use of a GAN-based framework with augmented data for PA image reconstruction from band-limited and limited view data. Specifically, we used open-source pix2pix GAN with augmented sinogram and time-reversal based reconstructed images as input and trained the network against the ground truth images. We present our results on simulated vascular images and in vivo data.

\section{MATERIALS AND METHODS}

We used the open-source GAN framework pix2pix for this work. ${ }^{14}$ Figure 1 shows a general block diagram of the GAN architecture with a generator and discriminator. Augmented data was used as the input to the generator and trained with output as ground truth image. The discriminator checks whether the image is from the actual ground truth image without artifacts or a generated image with artifacts. We modified the network to incorporate the augmented data. A convolutional layer followed by concatenation was added to the network. The generator had a UNet like architecture with skip connections. The discriminator is a deep convolutional neural network for classification between images with or without artifacts.

Most of the PA applications involve imaging blood vessels. Therefore we have chosen a blood vessel database,

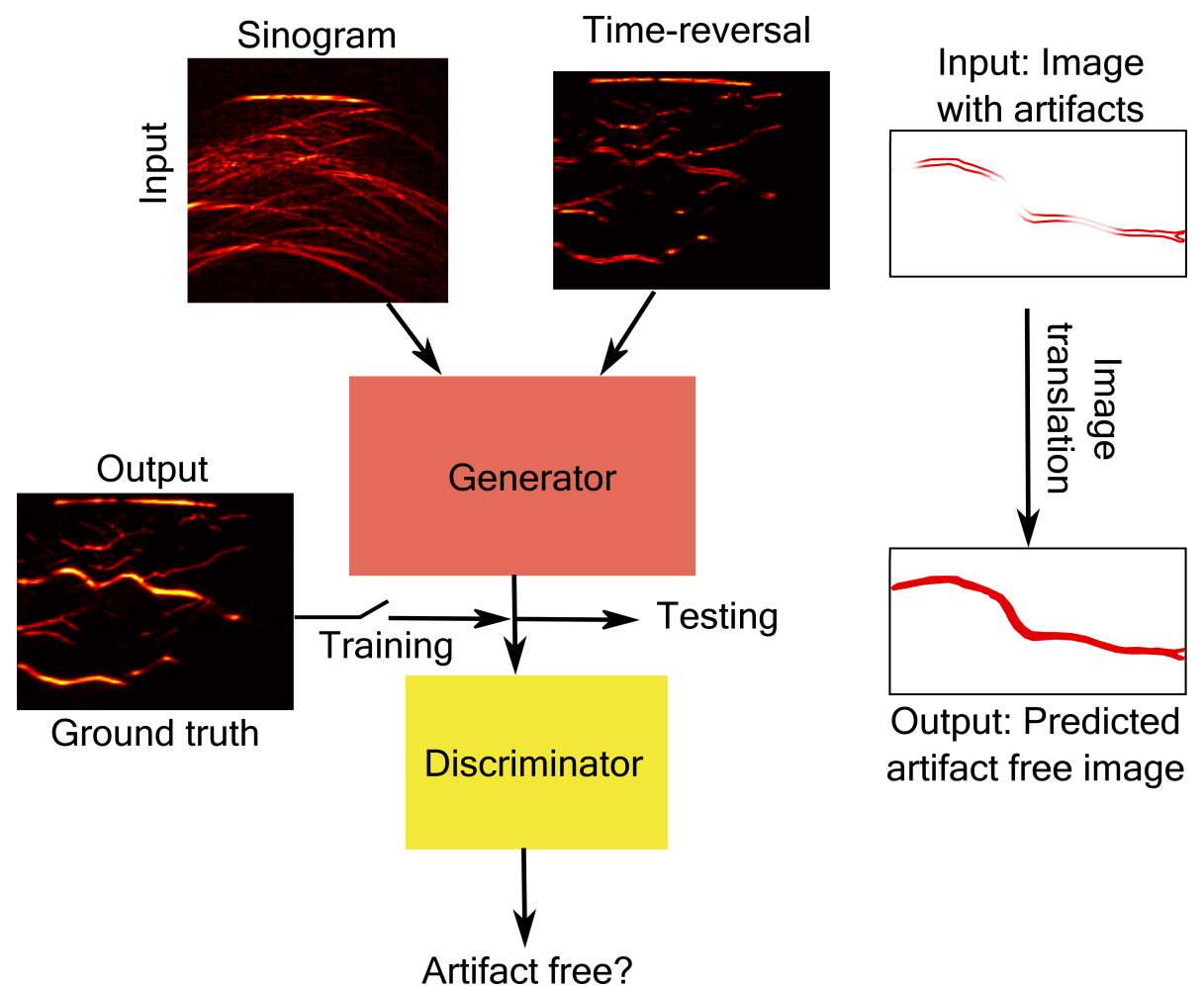

Figure 1. Generative Adversarial Networks (GANs) with augmented sinogram and reconstructed data as input for photoacoustic image enhancement. The goal is to perform image translation to enhance photoacoustic images and to remove artifacts.

Digital Retinal Images for Vessel Extraction (DRIVE). ${ }^{15}$ The dataset was then expanded by dividing each image 
into four images of size, $256 \times 256$. The dataset was further expanded by adding rotations to each image. Images generated by the handheld probe will also involve a skin layer. Hence, we also added a skin layer to all the images in the dataset. A total of 241 images were used in the study, and 48 images were used for testing.

The acoustic simulations were performed using the k-Wave toolbox in MATLAB. ${ }^{16}$ A $512 \times 512$ grid was used for the simulation. The images were scaled to the grid size to form the initial pressure. A linear array of 128 elements with a pitch of $0.315 \mathrm{~mm}$ was defined as the acoustic detector. A Gaussian bandpass filter with a center frequency of $7 \mathrm{MHz}$ and a bandwidth of $80 \%$ was used to mimic the bandlimited nature of the transducer. Further, the finite element size introduces a directivity to the transducer. A homogeneous medium with a speed of sound 1500 $\mathrm{m} / \mathrm{s}$ and a density of $1000 \mathrm{~kg} / \mathrm{m}^{3}$ were used in the simulation along with a power-law based acoustic attenuation $\left(0.75 \mathrm{~dB} / \mathrm{MHz}^{1.5} \mathrm{~cm}\right)$. The forward acoustic wave propagation using the $\mathrm{k}$-Wave toolbox was performed, and the time data (sinogram) was detected by the transducers. To mimic noise in the measurement, white noise with a signal-to-noise ratio of $30 \mathrm{~dB}$ was added to the sinogram. Time reversal algorithm was used to reconstruct the $\mathrm{PA}$ image and then resized to $256 \times 256$ pixels.

In vivo measurements were performed by LED-based PA imaging system AcousticX (Cyberdyne Inc., Japan). ${ }^{17}$ A linear transducer array having 128 elements and a pitch of $0.315 \mathrm{~mm}$ with a center frequency of $7 \mathrm{MHz}$ with $80 \%$ bandwidth. Two LED arrays of 144 elements each were used as the light source. The radial artery in the finger of a healthy volunteer was imaged. RF data from the system was obtained and the image was reconstructed using time reversal. The sinogram and the reconstructed image fed into the network to generate an artifact-free image.

\section{RESULTS AND DISCUSSION}

Results from simulated blood vessel phantom and in vivo and the predicted image are presented in this section. Figure 2 shows the results from simulated blood vessels. Figure 2a is the ground truth image, and Fig. 2b is from the time-reversal based reconstruction. The generated image using the trained network is shown in Fig. 2c. The error between the ground truth and the predicted image is shown in Fig. 2d. Figure 2e shows a line profile (green line in Fig. 2a) comparing PA amplitude between the ground truth, time-reversal reconstruction, and the prediction. In Fig. 2b, the effect of the transducer's bandlimited nature is evident with the double-wall in larger blood vessels. The lower signal level on structures oriented away from the transducer (on the top) is due to the directivity of the transducer. The predicted image is close to the ground truth image, and error in Fig. 2d shows high magnitude mostly at the intersection of blood vessels.

Figure 3a shows the time-reversal reconstructed image. The image consists of the skin layer and the radial artery. The GAN predicted PA image shows an enhancement of both skin layer and blood vessel. Although the image quality improved, the blood vessel is not reconstructed with uniform intensity. Further, there are no direct ways to compare the predicted image quality in the in vivo case. Cycle consistency may be a possibility to quantify the predictions in the in vivo case. ${ }^{18}$

Although this work shows promise in using GANs for PA image quality improvement, there are some limitations. In this work, we were limited in the number of images for training. Further, it was difficult to obtain blood vessel images that are close to the in vivo case. Future work will focus on including more images in the dataset and having a dataset as close as to the in vivo case. We will also focus on developing methods to quantify the in vivo results.

\section{CONCLUSION}

Results show that generative adversarial networks with augmented data can help to improve the image quality in photoacoustic imaging. Specifically, we have shown that the bandlimited and limited view artifacts can be removed using this method.

\section{ACKNOWLEDGMENTS}

We acknowledge funding from the 4TU federation in the precision medicine program. 


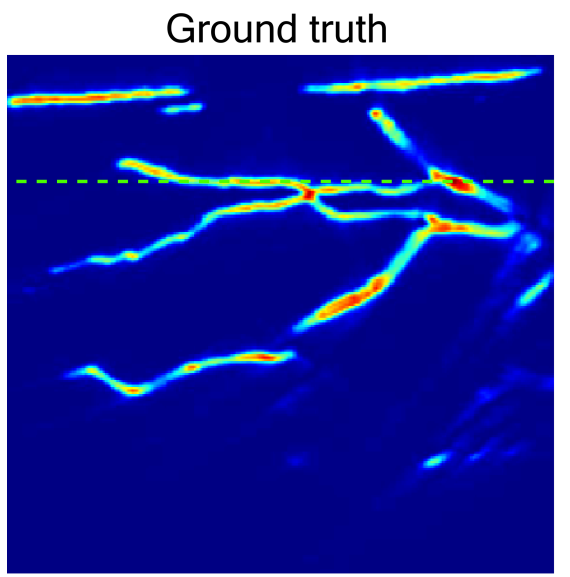

(a)

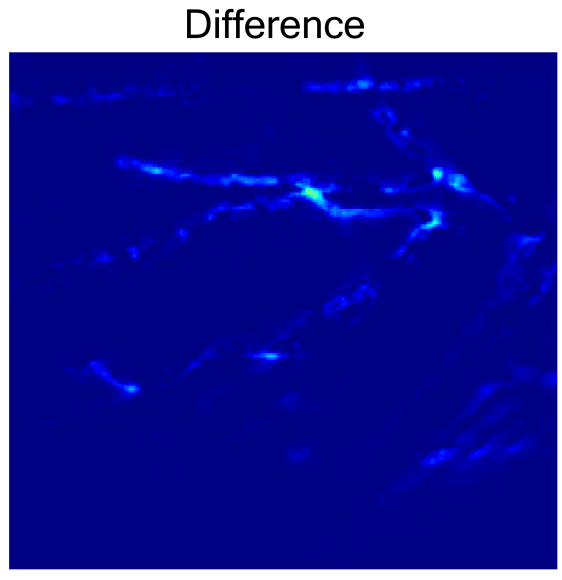

(d)

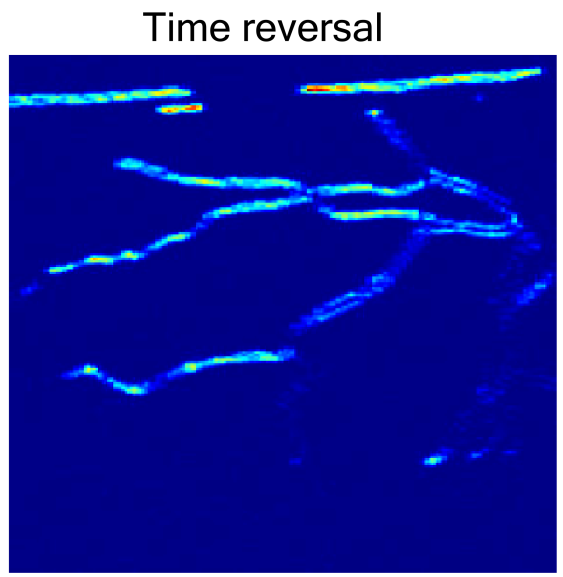

(b)

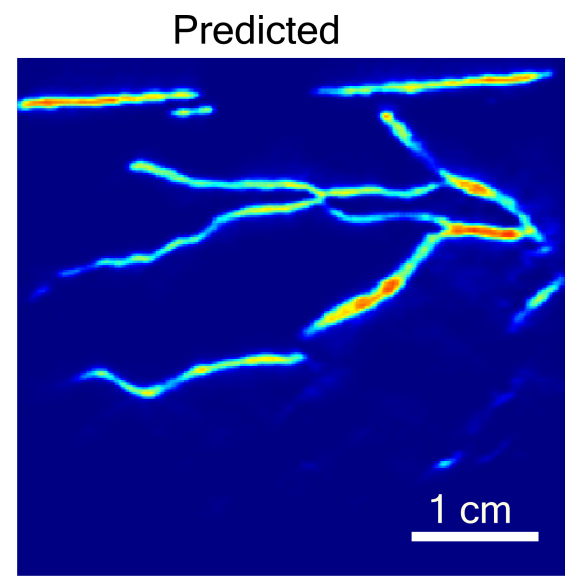

(c)

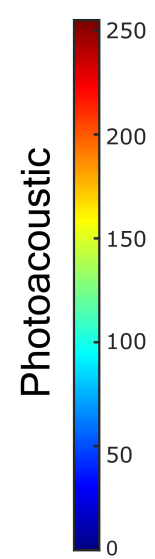

50
0

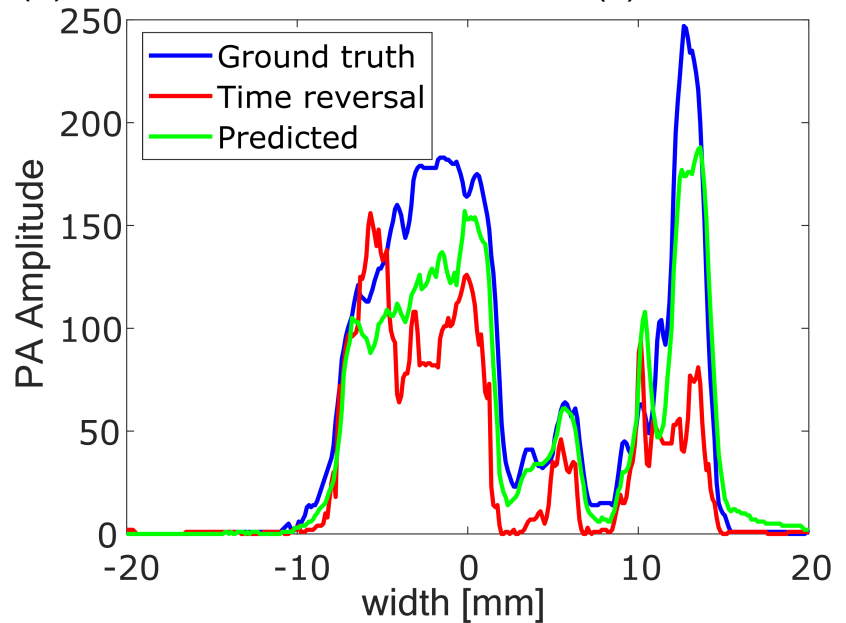

(e)

Figure 2. Simulated blood vessel phantom imaging. (a) Ground truth image. (b) Time-reversal reconstructed image. (c) GANs predicted image. (d) Difference between the ground truth and the prediction. (e) Line profile comparing the reconstruction methods. 


\section{Time reversal}

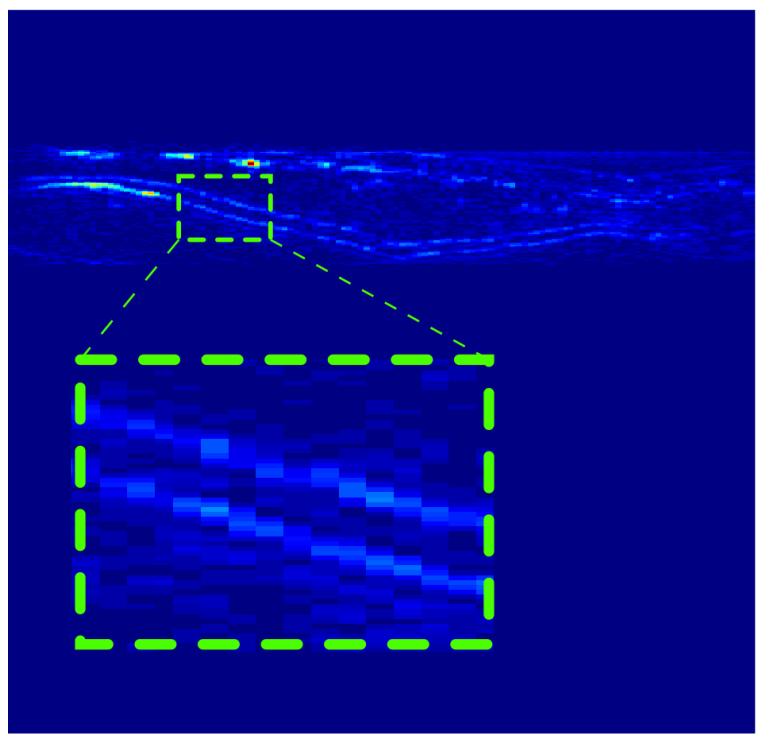

(a)

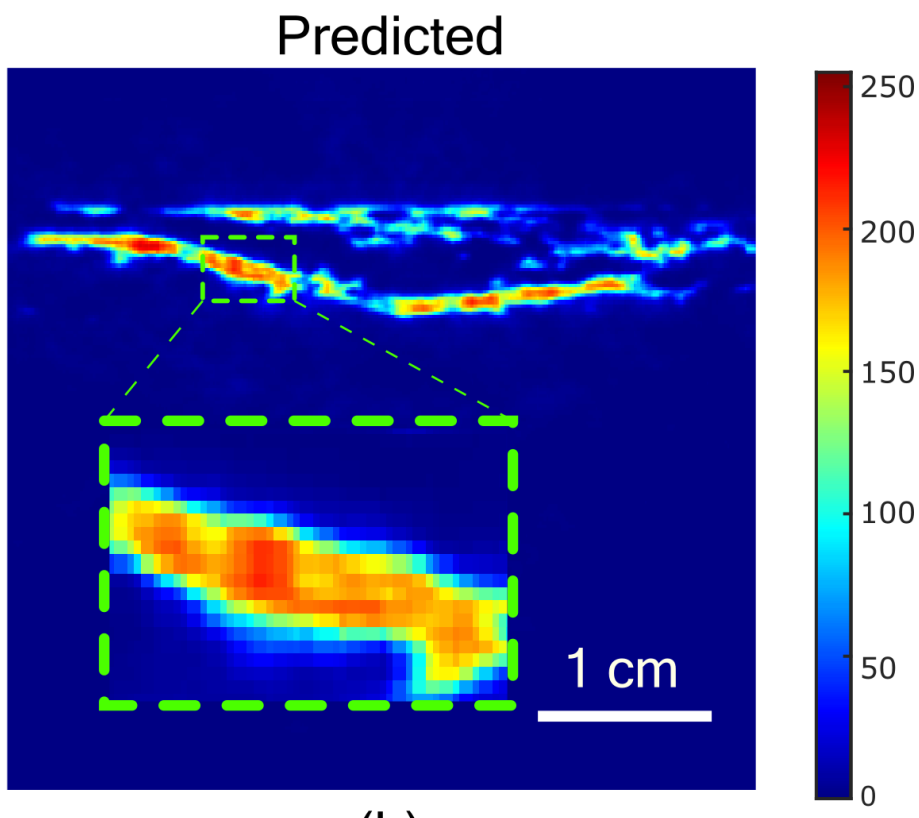

(b)

Figure 3. In vivo imaging. (a) Reconstruction with time reversal and (b) GANs predicted image.

\section{REFERENCES}

[1] Xu, M. and Wang, L. V., "Photoacoustic imaging in biomedicine," Review of Scientific Instruments 77(4), 041101 (2006).

[2] Singh, M. K. A., Steenbergen, W., and Manohar, S., "Handheld probe-based dual mode ultrasound/photoacoustics for biomedical imaging," Frontiers in Biophotonics for Translational Medicine , 209247 (2016).

[3] Ku, G., Wang, X., Stoica, G., and Wang, L. V., "Multiple-bandwidth photoacoustic tomography," Physics in Medicine ES Biology 49(7), 1329 (2004).

[4] Francis, K. J., Boink, Y. E., Dantuma, M., Singh, M. K. A., Manohar, S., and Steenbergen, W., "Tomographic imaging with an ultrasound and led-based photoacoustic system," Biomedical Optics Express 11(4), 2152-2165 (2020).

[5] Francis, K. J., Chinni, B., Channappayya, S. S., Pachamuthu, R., Dogra, V. S., and Rao, N., "Multiview spatial compounding using lens-based photoacoustic imaging system," Photoacoustics 13, 85-94 (2019).

[6] Xu, Y., Wang, L. V., Ambartsoumian, G., and Kuchment, P., "Reconstructions in limited-view thermoacoustic tomography," Medical Physics 31(4), 724-733 (2004).

[7] Hauptmann, A., Lucka, F., Betcke, M., Huynh, N., Adler, J., Cox, B., Beard, P., Ourselin, S., and Arridge, S., "Model-based learning for accelerated, limited-view 3-d photoacoustic tomography," IEEE Transactions on Medical Imaging 37(6), 1382-1393 (2018).

[8] Deng, H., Wang, X., Cai, C., Luo, J., and Ma, C., "Machine-learning enhanced photoacoustic computed tomography in a limited view configuration," Advanced Optical Imaging Technologies II 11186, 111860J (2019).

[9] Waibel, D., Gröhl, J., Isensee, F., Kirchner, T., Maier-Hein, K., and Maier-Hein, L., "Reconstruction of initial pressure from limited view photoacoustic images using deep learning," Photons Plus Ultrasound: Imaging and Sensing 2018 10494, 104942S (2018).

[10] Davoudi, N., Deán-Ben, X. L., and Razansky, D., "Deep learning optoacoustic tomography with sparse data," Nature Machine Intelligence 1(10), 453-460 (2019).

[11] Awasthi, N., Jain, G., Kalva, S. K., Pramanik, M., and Yalavarthy, P. K., "Deep neural network-based sinogram super-resolution and bandwidth enhancement for limited-data photoacoustic tomography," IEEE Transactions on Ultrasonics, Ferroelectrics, and Frequency Control 67(12), 2660-2673 (2020). 
[12] Singh, M. K. A., Sivasubramanian, K., Sato, N., Ichihashi, F., Sankai, Y., and Xing, L., "Deep learningenhanced led-based photoacoustic imaging," Photons Plus Ultrasound: Imaging and Sensing 2020 11240, $1124038(2020)$.

[13] Vu, T., Li, M., Humayun, H., Zhou, Y., and Yao, J., "A generative adversarial network for artifact removal in photoacoustic computed tomography with a linear-array transducer," Experimental Biology and Medicine 245(7), 597-605 (2020).

[14] Isola, P., Zhu, J.-Y., Zhou, T., and Efros, A. A., "Image-to-image translation with conditional adversarial networks," Proceedings of the IEEE Conference on Computer Vision and Pattern Recognition, 1125-1134 (2017).

[15] Staal, J., Abràmoff, M. D., Niemeijer, M., Viergever, M. A., and Van Ginneken, B., "Ridge-based vessel segmentation in color images of the retina," IEEE Transactions on Medical Imaging 23(4), 501-509 (2004).

[16] Treeby, B. E. and Cox, B. T., "k-wave: Matlab toolbox for the simulation and reconstruction of photoacoustic wave fields," Journal of Biomedical Optics 15(2), 021314 (2010).

[17] Bulsink, R., Kuniyil Ajith Singh, M., Xavierselvan, M., Mallidi, S., Steenbergen, W., and Francis, K. J., "Oxygen saturation imaging using led-based photoacoustic system," Sensors 21(1), 283 (2021).

[18] Hauptmann, A. and Cox, B. T., "Deep learning in photoacoustic tomography: Current approaches and future directions," Journal of Biomedical Optics 25(11), 112903 (2020). 